\title{
The Flavour of Gravity
}

\author{
R Delbourgo $\dagger$ \\ † School of Mathematics and Physics, University of Tasmania, Locked Bag 37 GPO \\ Hobart, AUSTRALIA 7001 \\ E-mail: Bob.Delbourgo@utas.edu.au
}

\begin{abstract}
By attaching five (complex) anticommuting property coordinates to the four (real) commuting space-time ones, it is possible to accommodate all the known fundamental particles in their three generations. A general relativistic extension to space-time-property can be carried out such that the gauge fields find their place in the space-property sector and the Higgs scalars in the property-property sector. The full curvature is the sum of the gravitational curvature, the gauge field Lagrangian and the Higgs field contribution; property curvature may be linked to the cosmological term.
\end{abstract}

Submitted to: J. Phys. A: Math. Gen.

PACS numbers: $11.10 \mathrm{Kk}, 11.30 . \mathrm{Hv}, 11.30 . \mathrm{Pb}, 12.10 .-\mathrm{g}$ 


\section{Property coordinates}

The roots of a grand unified theory of everything can be traced to Einstein's search for a unification of gravity with electromagnetism. That quest led Einstein to consider space-time schemes in which additional coordinates were appended to three space and one time coordinate, and to other initiatives in which the gravitational metric was no longer a symmetric tensor. These concepts have their modern counterparts in respect of strings/branes existing in enlarged space-times and in extensions which allow for noncommuting coordinates. The last 30 years have spawned a lot of developments along these lines, together with the incorporation of supersymmetry. Despite the intensity of these investigations and their great beauty it must admitted that there is no firm experimental evidence for their realization in nature; all that we observe today is described by a three-generation standard model with its multiplicity of parameters, with no evidence of spartners and no sign of higher dimensions. It is a rather unfortunate conclusion and suggests that one must look to other ways of unifying the natural forces, if that is our ultimate goal.

Over the last several years, with a number of collaborators [1, we have studied an alternative approach which smacks of supersymmetry, but is somewhat different. The basic idea is to append anticommuting coordinates $\zeta$ to space-time $x$; unlike standard supersymmetry these coordinates do not carry spin but instead are Lorentz scalar - like the BRST fields used to implement unitarity/gauge-fixing in quantized gauge theories. Although this may seem somewhat unnatural we do not run into the problems of higher spins connected with extended supersymmetries and this is a definite bonus. We are led to ascribe 'property' to the various $\zeta$. Due to anticommutativity, finite polynomials in $\zeta$ will lead to a finite set of composite properties; this is in contrast to string-like excitations associated with bosonic higher dimensions that produce an infinite number of modes. An intriguing aspect of such a formulation is that fermionic coordinates effectively reduce [2] the number of dimensions, so that one might contemplate a universe with zero net dimensions, like it was before the big bang one presumes. The main lesson of supersymmetry, that provided one balances the number of bosons and fermions (with equal source couplings), one can eliminate the worst of the infinities of conventional field theory, thereby obtaining a naturally fine-tuned theory, is another important facet.

These are the grounds which motivate the present paper. In Section 2, we recapitulate the reasons for attaching five complex property coordinates to spacetime (see also Appendix B); the principal consequences in relation to the fundamental particle spectrum are detailed there. The really new work lies in Section 3; it concerns the general relativistic extension of the work so as to include all the force fields via an extended metric, where the separation describes the difference in two events in location and in property. In order to make this generalization it is essential to get all the sign factors correct when constructing tensors and forming invariants and the like over commuting and anticommuting variables. In an earlier paper this was patched somewhat haphazardly by including torsion, but here we do the analysis properly in Appendix A. 
Because the algebra becomes very difficult when tackling five $\zeta$ in full nonlinear glory, we shall outline the results for two simplified metrics which give a foretaste of expectations for the complete case. Both these examples, explored in Section 4, use just one complex $\zeta$ or a real pair $\xi, \eta$. The first example corresponds to curved property but flat space-time while the second example incorporates electromagnetism and leaves the property sector flat. We find that property curvature has an impact on the cosmological term, while the connection between space and property leads quite naturally to the gravitational plus electromagnetic Lagrangian, the latter following from other components of the full Riemann-Ricci tensor. In future work we intend to amalgamate all these ideas and use the entire $\zeta$ set.

\section{Superfields}

To gain some perspective on property, or flavour as it was once called, we start off with an elementary example involving two anticommuting complex coordinates $\zeta^{1}, \zeta^{2}$ which are appended to two-dimensional space-time $x^{0}, x^{1}$. Let $\zeta^{1}$ correspond to 'electronicity' and $\zeta^{2}$ to 'protonicity', associated with charge and fermion number assignments $Q\left(\zeta^{1}, \zeta^{2}\right)=(-1,1)$ and $F\left(\zeta^{1}, \zeta^{2}\right)=(1,1)$ respectively. Superfields are functions of $x$ and $\zeta, \bar{\zeta}$ and can be expanded in $\zeta$-polynomials. To avoid conflict with spin-statistics one connects bosons with even powers of $\zeta$ and fermions with odd powers of $\zeta$. Therefore let $\Phi$ be a real superBose field describing bosons having the expansion

$$
\Phi(x, \zeta, \bar{\zeta})=A(x)+\bar{C}(x) \zeta^{1} \zeta^{2}+\bar{\zeta}_{2} \bar{\zeta}_{1} C(x)+\bar{\zeta}_{\mu} D^{\mu}{ }_{\nu}(x) \zeta^{\nu}+\bar{\zeta}_{2} \bar{\zeta}_{1} \zeta^{1} \zeta^{2} F(x) \cdot(1)
$$

The coefficient fields $A, C, F$ and two of the $D$ are charge neutral, but two of the $D$ are doubly charged. We can regard the $C$ with their polynomials $\zeta^{1} \zeta^{2}$ as connoting (composite) atomicity (like $e p, \bar{e} \bar{p}$ ) while the two neutral $D$ may be associated with mesonicity (like $e \bar{e}, p \bar{p}$ ). The doubly charged $D$, tied to $\zeta^{1} \bar{\zeta}^{2}, \zeta^{2} \bar{\zeta}^{1}$ (like $e \bar{p}, p \bar{e}$ ) are more problematic because they do not correspond to physically realized states. We can carry out the same construction for fermions, of some chirality,

$$
\Psi(x, \zeta, \bar{\zeta})=B_{\mu}^{(c)}(x) \zeta^{\mu}+\bar{\zeta}_{\mu} B^{\mu}(x)+\bar{\zeta}_{\nu} \zeta^{\nu} E_{\mu}^{(c)}(x) \zeta^{\mu}+\bar{\zeta}_{\nu} \zeta^{\nu} \bar{\zeta}_{\mu} E^{\mu}(x)
$$

where $B_{1}, B_{2}$ connote $(e, p)$ states and $E_{1}, E_{2}$ might describe another generation of $(e, p)$ or excited versions. It should be stressed that because $\Psi$ is overall commuting, the component fields $B$ and $E$ will anticommute; they carry spinor labels, like $\Psi$, which have been deliberately suppressed.

However by invoking duality (see Appendix $\mathrm{C}$ for the detailed five $\zeta$ case) we can roughly halve the number of components and eliminate some unwanted states. It is important to realize that duality $\left(^{\times}\right)$has no effect on the charge and fermion number assignments. Therefore its imposition does not affect quantum numbers and can be used at will. (Also a double duality operation coincides with the identity.) Specifically,

$$
\begin{aligned}
& (1)^{\times}=\left(\bar{\zeta}_{\nu} \zeta^{\nu}\right)^{2} / 2 !,\left(\zeta^{\mu}\right)^{\times}=\zeta^{\mu}\left(\bar{\zeta}_{\nu} \zeta^{\nu}\right),\left(\zeta^{1} \zeta^{2}\right)^{\times}=-\zeta^{1} \zeta^{2} \\
& \left(\bar{\zeta}_{1} \zeta^{1}\right)^{\times}=\bar{\zeta}_{2} \zeta^{2}, \quad\left(\bar{\zeta}_{1} \zeta^{2}\right)^{\times}=-\bar{\zeta}_{1} \zeta^{2} \quad\left(\bar{\zeta}_{2} \zeta^{1}\right)^{\times}=-\bar{\zeta}_{2} \zeta^{1}
\end{aligned}
$$


plus the conjugate relations $(\zeta \leftrightarrow \bar{\zeta})$ and duals. By insisting that $(\Phi, \Psi)^{\times}=(\Phi, \Psi)$ are both self-dual we can satisfyingly dispose of the doubly charged states as well as $C$, relate $F$ to $A$ and $E$ to $B$. What survives are the self-dual

$$
\Phi=\left(A+S \bar{\zeta}_{\mu} \zeta^{\mu}\right)\left(1+\bar{\zeta}_{\nu} \zeta^{\nu}\right) / 2, \quad \Psi=\left(\bar{\zeta}_{\mu} B^{\mu}+B_{\mu}^{(c)} \zeta^{\mu}\right)\left(1+\bar{\zeta}_{\nu} \zeta^{\nu}\right) / 2
$$

A nice description of all this is to picture the expansion terms via a magic square, whose rows and columns are labelled by the powers of $\zeta$ and $\bar{\zeta}$ as they occur in the series (1) and (2). Fermions fit into the odd label row/columns and the bosons in the even label row/columns; conjugation corresponds to reflection about the main diagonal while duality corresponds to reflection about the cross-diagonal. By placing the fermions and bosons in the same square for convenience (but not implying that they have the same Lorentz transformation properties) we can understand the diminution of the magic square as a consequence of cross-reflection:

$$
\left(\begin{array}{ccc}
A & B & C \\
B^{(c)} & D & E \\
\bar{C} & E^{(c)} & F
\end{array}\right) \rightarrow_{\text {self-duality }}\left(\begin{array}{ccc}
A & B & 0 \\
B^{(c)} & S & B \\
0 & B^{(c)} & A
\end{array}\right) .
$$

We can then go on to construct free and interaction Lagrangians for those superfields, where the masses arise from expectation values $s, a$, and so on. We shall not elaborate on this as the procedure for doing this is very straightforward; anyway the more realistic case of five $\zeta$ will be described in some detail presently. The main thrust of this section is that properties such as flavours, colours, charges, generation number, etc. can be built up compositely and that these are finite. The resulting states which may be more numerous than one would wish can then be culled by imposing duality constraints on the superfields which comprise them all.

The basic aim of the proposed scheme is to accommodate all known fundamental particles simply and naturally without special pleading for symmetry groups and particular representations or repetition number. Of course we are inspired by the monumental work done on Grand Unified Theories [3] in guiding us towards this end over the last few decades; more specifically it will come as no surprise that one is directed to the most economical (and therefore the most popular) proposals - those built upon $\mathrm{SU}(5)$ and $\mathrm{SO}(10)$ algebras. In constructing the model, we would like to feature colour, charge, fermion number, flavour and generation when building up properties from the basic $\zeta$. To be sure many prequark schemes have been devised [4, but we wish to base our formulation entirely in terms of anticommuting variables without worrying unduly about what particular dynamics favours the states seen in nature.

Three $\zeta$ in the form of a charge $Q=-1 / 3$ colour triplet (down-type quark) fall well short of our goal, for whereas $\zeta^{1} \zeta^{2} \zeta^{3}$ might serve for a lepton there is no room for a neutrino or up-type quark, and anyhow where are all the other generations? In Appendix B we prove that four $\zeta$ are also insufficient, so this leads us to the next case, $N=5$ which, coincidentally, accords with popular choice. A first quantized version of this model was considered by Jarvis, Dondi and White [5]; here we shall examine a 
second quantized version in greater depth. So let us quickly summarize the consequences of using property coordinates possessing $Q\left(\zeta^{0}, \zeta^{1}, \zeta^{2}, \zeta^{3}, \zeta^{4}\right)=(0,1 / 3,1 / 3,1 / 3,-1)$ and fermion number $F\left(\zeta^{0}, \zeta^{1}, \zeta^{2}, \zeta^{3}, \zeta^{4}\right)=(1,-1 / 3,-1 / 3,-1 / 3,1)$. [Incidentally the sum of $Q$ vanishes which helps in anomaly cancellation.] We may ascribe 'neutrinicity' to $\zeta^{0}$, 'chromicity' to $\zeta^{i}$ where $i=1,2,3$ are the three colours, and charged 'leptonicity' to $\zeta^{4}$ in building up properties/flavours.

As before, we follow through the property expansions of the (overall) Bose superfields to see what emerges, and in particular we look out for repetitions of leptons, neutrinos, coloured up-quarks and down-quarks:

$$
\begin{aligned}
& \Phi(x, \zeta, \bar{\zeta})=\sum_{\text {even } r+\bar{r}}(\bar{\zeta})^{\bar{r}} \phi_{(\bar{r}),(r)}(\zeta)^{r} \\
& \Psi_{\alpha}(x, \zeta, \bar{\zeta})=\sum_{\text {odd } r+\bar{r}}(\bar{\zeta})^{\bar{r}} \psi_{\alpha(\bar{r}),(r)}(\zeta)^{r} .
\end{aligned}
$$

The number of components of fermionic $\psi$ and bosonic $\phi$ each number 512 so they invite pruning. A primary way to do this is to tie reflection of the magic $6 \times 6$ square about the main diagonal to conjugation, or or

$$
\psi_{\alpha(\bar{r}),(r)}^{(c)}=\psi_{\alpha(r)(\bar{r})}, \quad \phi_{(\bar{r}),(r)}=\phi_{(r)(\bar{r})}^{\dagger} .
$$

But there are still too many states; so we apply a secondary condition that superfields are selfdual in some way, corresponding to reflection about the cross-diagonal. (The dual operation ${ }^{x}$ does not affect quantum numbers.) Before implementing duality let us point out some unwanted states hiding in the square, which duality might exorcise. The most embarrassing are the combinations $\bar{\zeta}_{0} \bar{\zeta}_{4} \zeta^{1} \zeta^{2} \zeta^{3}$ and $\bar{\zeta}_{4} \zeta^{0} \zeta^{1} \zeta^{2} \zeta^{3}$ which possess $F=3$ and $Q=2$ respectively - disposing of them would be a relief. Since by duality (see Appendix C),

$$
\left((\zeta)^{r}(\bar{\zeta})^{\bar{r}}\right)^{\times}=(\zeta)^{5-\bar{r}}(\bar{\zeta})^{5-r},
$$

it pays to make the superfield $\Psi$ anti-selfdual: $(\Psi)^{\times}=-\Psi$ or $\psi_{(\bar{r})(r)}=-\psi_{(5-r)(5-\bar{r})}$. This condition gets rid of the neutrino-like state in the upper right corner too, $\zeta^{0} \zeta^{1} \zeta^{2} \zeta^{3} \zeta^{4}$. We assume that the same constraint can be imposed on the Bose superfield. One good thing is that duality automatically provides a term containing the product $\left(\bar{\zeta}_{\mu} \zeta^{\mu}\right)^{5} / 5$ ! in field products, so when integrating over the property coordinates $\int \prod_{\nu=1}^{5} d \zeta^{\nu} d \bar{\zeta}_{\nu}$ we are guaranteed to get nonzero answers by Berezin integration.

Many fermions survive the anti-selfduality constraint, including some that are colour sextets. However, like everyone else, we presume that asymptotic states must be colour singlets so we shall not fret about them; of more concern to us is to discover how many quarks and leptons remain so as to be able to count repetitions or possible generations. We shall therefore list below in a magic square all the relevant ones ${ }^{*}$ and - mean related by conjugation or duality respectively). They lie scattered in the odd sectors as shown: 


\begin{tabular}{|c|c|c|c|c|c|c|}
\hline$r \backslash \bar{r}$ & 0 & 1 & 2 & 3 & 4 & 5 \\
\hline 0 & & $L_{1}, N_{1}, D_{1}^{c}$ & & $L_{5}^{c}, D_{5}, U_{1}$ & & \\
\hline 1 & $*$ & & $L_{2,3}, N_{2,3}, D_{2,3,8}^{c}, U_{2}$ & & $L_{6}, D_{6}, U_{3}$ & \\
\hline 2 & & $*$ & & $L_{4}, N_{4}, D_{4,7}^{c}, U_{4}$ & & - \\
\hline 3 & $*$ & & $*$ & & - & \\
\hline 4 & & $*$ & & $*$ & & - \\
\hline 5 & $*$ & & $*$ & & $*$ & \\
\hline
\end{tabular}

It is apparent that there is room for four up-type quarks, eight down-type quarks plus six charged leptons and four neutrinos, so we can certainly accommodate the known three generations. The model predicts that there are some new down-quarks to be discovered and perhaps other charged leptons and neutrinos. (It does not predict the masses of these fermions until the Higgs expectation values are folded in through the Yukawa interactions.) We must also point out that the independent polynomials $\zeta^{0} \zeta^{4} \zeta^{i}$ and $\zeta^{0} \zeta^{4} \zeta^{i}\left(\bar{\zeta}_{j} \zeta^{j}\right)$ cannot be associated with the normal up-quarks because they carry $F=5 / 3$, if one sticks to the earlier fermion number assignments. It is hard to know what to make of these predictions. Having additional down-quarks without their upquark counterparts does not accord with the standard family picture; yet most persons would admit that the wide mass disparities within and across the three generations appears to make a mockery of the standard groupings. It is not impossible that new $D$-quarks may have some connection with the recently discovered pentaquarks states like $\Theta^{+} \sim u u d d \bar{s}$ and $\Xi^{--} \sim d d s s \bar{u}$, but until these resonances are firmly established the subject is probably not worth debating.

Next we turn to the scalar (Higgs) sector, which has the bosons sprinkled throughout even sectors of the magic square. Again we meet a plethora of states which can be somewhat reduced by invoking conjugation and duality. Of particular focus are the states which are neutral $(F=Q=0)$ since their expectation values can impart masses to the fermions. Nine such constants enter in principle:

- one $\phi_{(0)(0)}=\langle\phi\rangle$

- one $\phi_{(0)(4)}=\left\langle\phi_{1234}\right\rangle$

- three $\phi_{(1)(1)}=\left\langle\phi_{0}^{0}, \phi_{4}^{4}, \phi_{i}^{i}\right\rangle$

- four $\phi_{(2)(2)}=\left\langle\phi_{04}^{04}, \phi_{0 k}^{0 k}, \phi_{4 k}^{4 k}, \phi_{i j}^{i j}\right\rangle$,

others being related by duality. This is much fewer than the number of constants used in the standard model so we anticipate that some useful mass relations will pan out after one works out the mass values for the fermions - no easy task. To appreciate the severity of the complications, take the Yukawa interactions between the $U$-components of the superfield $\Psi$,

$\Psi \supset \epsilon^{i j k} \bar{\zeta}_{0} \bar{\zeta}_{i} \bar{\zeta}_{j} U_{1 k}\left(1+\bar{\zeta}_{4} \zeta^{4} \bar{\zeta}_{l} \zeta^{l}\right)+\bar{\zeta}_{0} \zeta^{4} \zeta^{k} U_{2 k}\left(1+\left(\bar{\zeta}_{l} \zeta^{l}\right)^{2} / 2\right)+\epsilon^{i j k} \bar{\zeta}_{0} \bar{\zeta}_{i} \bar{\zeta}_{j} U_{3 k}\left(\bar{\zeta}_{l} \zeta^{l}-\bar{\zeta}_{4} \zeta^{4}\right)+$ conj

and their interaction $\bar{\Psi}^{\alpha} \Psi_{\alpha} \Phi$ with the classical part of the anti-selfdual Higgs superfield,

$$
\langle\Phi\rangle=\langle\phi\rangle\left[1-\left(\bar{\zeta}_{\mu} \zeta^{\mu}\right)^{5} / 5 !\right]+\left\langle\phi_{1234}\right\rangle\left[\zeta^{1} \zeta^{2} \zeta^{3} \zeta^{4}-\bar{\zeta}_{1} \bar{\zeta}_{2} \bar{\zeta}_{3} \bar{\zeta}_{4}\right]\left[1-\bar{\zeta}_{0} \zeta^{0}\right]+
$$


Flavour of Gravity

$$
\begin{aligned}
& \left\langle\phi_{0}^{0}\right\rangle\left[\bar{\zeta}_{0} \zeta^{0}-\bar{\zeta}_{4} \zeta^{4}\left(\bar{\zeta}_{i} \zeta^{i}\right)^{3} / 3 !\right]+\left\langle\phi_{4}^{4}\right\rangle\left[\bar{\zeta}_{4} \zeta^{4}-\bar{\zeta}_{0} \zeta^{0}\left(\bar{\zeta}_{i} \zeta^{i}\right)^{3} / 3 !\right]+ \\
& \left\langle\phi_{04}^{04}\right\rangle\left[\bar{\zeta}_{0} \zeta^{0} \bar{\zeta}_{4} \zeta^{4}-\left(\bar{\zeta}_{i} \zeta^{i}\right)^{3} / 3 !\right]+\left\langle\phi_{n}^{n}\right\rangle\left[\bar{\zeta}_{k} \zeta^{k}-\bar{\zeta}_{0} \zeta^{0} \bar{\zeta}_{4} \zeta^{4} \bar{\zeta}_{i} \zeta^{\zeta^{\prime}} \bar{\zeta}_{j} \zeta^{j} / 2\right]+ \\
& \left\langle\phi_{0 n}^{0 n}\right\rangle\left[\bar{\zeta}_{k} \zeta^{k} \bar{\zeta}_{0} \zeta^{0}-\bar{\zeta}_{4} \zeta^{4} \bar{\zeta}_{i} \zeta^{i} \bar{\zeta}_{j} \zeta^{j}\right]+\left\langle\phi_{4 n}^{4 n}\right\rangle\left[\bar{\zeta}_{k} \zeta^{k} \bar{\zeta}_{4} \zeta^{4}-\bar{\zeta}_{0} \zeta^{0} \bar{\zeta}_{i} \zeta^{i} \bar{\zeta}_{j} \zeta^{j} / 2\right]+ \\
& \left\langle\phi_{m n}^{m n}\right\rangle\left[\bar{\zeta}_{i} \zeta^{i} \bar{\zeta}_{j} \zeta^{j} / 2-\bar{\zeta}_{k} \zeta^{k} \bar{\zeta}_{0} \zeta^{0} \bar{\zeta}_{4} \zeta^{4}\right],
\end{aligned}
$$

ignoring normalization factors. We obtain the combinations

$$
\begin{gathered}
\langle\phi\rangle\left(\bar{U}_{1} U_{1}+\bar{U}_{2} U_{2}+\bar{U}_{3} U_{3}\right)+\left\langle\phi_{1234}\right\rangle\left(\bar{U}_{2} U_{1}+\bar{U}_{1} U_{2}\right)+\left\langle\phi_{4}^{4}\right\rangle\left(\bar{U}_{3} U_{1}+\bar{U}_{1} U_{3}\right)+ \\
\left\langle\phi_{l}^{l}\right\rangle\left(\bar{U}_{2} U_{2}+\bar{U}_{3} U_{1}+\bar{U}_{1} U_{3}\right)+\left\langle\phi_{4 l}^{4 l}\right\rangle \bar{U}_{1} U_{1}+\left\langle\phi_{i j}^{i j} \bar{U}_{2} U_{2}\right.
\end{gathered}
$$

that need to be diagonalized. The $D$ quarks and $L, N$ involve other expectation values and mixings which must also be unravelled to arrive at the final masses of physical states. This is a nontrivial problem which is left for future research, but with only nine $\langle\phi\rangle$, intriguing consequences may unfold.

\section{Gauge fields and general relativity with property}

What is the the role of gauge fields in this scenario? One could mimic the practice of supersymmetry and construct some sort of vector superfield in which the gauge fields are embedded, but we prefer to ape the Klein-Kaluza picture where gauge fields connect space-time with the extended coordinates: in our description these are anticommuting and connected with property. Because the gauge fields are the carriers of forces connected with characteristic charge or colour it is perfectly natural to use them as links between $x$ and $\zeta$. A general relativistic framework comes to mind therefore in which the extended metric provides the separation between two events in location and in type. We may imagine a generalized coordinate $X^{M}=\left(x^{m}, \zeta^{\mu}\right)=\left(x^{m}, \xi^{\mu}, \eta^{\mu}\right)$ which combines position and property, such that a generalized squared distance between two coordinates can be simply expressed in the Hermitian form (here $\bar{\zeta}^{\bar{\mu}} \equiv \bar{\zeta}_{\mu}$ of the previous section as we want to use general relativistic notation rather than particle physics notation):

$d s^{2}=d X^{N} d X^{M} G_{M N}=d x^{m} d x^{n} G_{m n}+2 d x^{m} d \zeta^{\nu} G_{\nu m}+2 d x^{m} d \bar{\zeta}^{\bar{\nu}} G_{\bar{\nu} m}+2 d \bar{\zeta}^{\bar{\mu}} d \zeta^{\nu} G_{\nu \bar{\mu}}$.

The space-space components are to be associated with gravity, the space-property components are to contain the gauge fields and the property-property components comprise the Higgs scalars. Since $d s^{2}$ is real, note that $G_{m n}$ is bosonic and symmetric, $G_{\mu \nu}$ is bosonic and antisymmetric, while $G_{m \nu}$ and $G_{\bar{\mu} n}$ are symmetric but fermionic. Thus the ordering of labels and fields is of crucial importance in ensuring the correctness of the algebra - the price to be paid for merging bosonic $x$ with fermionic $\xi, \eta$.

The gauge fields occur in the fermionic sectors $G_{n \mu}$ as we shall shortly see, but before placing them, observe that a length scale $\Lambda$ is obligatory when combining dimensionless $\zeta$ to dimensionful $x$. Thus consider a typical metric that arises from a vielbein $E_{M}^{A}$ having components,

$$
\left(\begin{array}{cc}
e_{m}^{a} & i \Lambda\left(A_{m}\right)_{\mu}^{\alpha} \zeta^{\mu} \\
0 & \Lambda \delta_{\mu}^{\alpha}
\end{array}\right)
$$


producing the hermitian form

$d s^{2}=d x^{m} d x^{n} g_{n m}+2 \Lambda^{2}\left[d \bar{\zeta}^{\bar{\mu}}-i d x^{m} \bar{\zeta}^{\bar{\kappa}}\left(A_{m}\right)_{\bar{\kappa}}^{\bar{\mu}}\right] \delta_{\bar{\mu} \nu}\left[d \zeta^{\nu}+i d x^{n}\left(A_{n}\right)_{\lambda}^{\nu} \zeta^{\lambda}\right], \quad g_{m n}=e_{m}^{a} e_{n}^{b} \eta_{a b} \cdot(7)$

The introduced scale $\Lambda$ may or may not be associated with the Planck length (or Newton's constant); we leave this open for the moment. Note too that there is no comfortable place for a gravitino in this picture as it would be tied with a fermionic field and does not sit well in the same sector as $A$, unless one introduces an extra spin property quartet, which one is loth to do.

The differential combination $\left(d \zeta+i d x^{n} A_{n} \zeta\right)$, which is very like the $5 \mathrm{D} \mathrm{K}-\mathrm{K}$ scenario, suggests how gauge transformations come about. Consider the simple coordinate change that mixes up the properties but leaves space-time intact,

$$
x \rightarrow x^{\prime}=x, \quad \zeta \rightarrow \zeta^{\prime}=\exp [i \Theta(x)] \zeta, \quad \bar{\zeta} \rightarrow \bar{\zeta}^{\prime}=\bar{\zeta} \exp [-i \Theta(x)]
$$

and look at the transformation property of $G_{m \zeta}=i \Lambda^{2} \bar{\zeta} A_{m}$ (see Appendix A):

$$
G_{m \zeta}(X)=\frac{\partial X^{\prime R}}{\partial x^{m}} \frac{\partial X^{\prime S}}{\partial \zeta} G_{S R}^{\prime}\left(X^{\prime}\right)(-1)^{[R]}=\frac{\partial \zeta^{\prime}}{\partial \zeta} G_{\zeta m}^{\prime}-\frac{\partial \bar{\zeta}^{\prime}}{\partial x^{m}} \frac{\partial \zeta^{\prime}}{\partial \zeta} G_{\zeta \bar{\zeta}}^{\prime}
$$

Factoring out $i \Lambda^{2}$ this leads to

$$
\bar{\zeta} A_{m}=\bar{\zeta}^{\prime} A_{m}^{\prime} \exp [i \Theta(x)]-i \bar{\zeta}\left(\partial_{m} \exp [-i \Theta(x)]\right) \exp [i \Theta(x)]
$$

which, as one would wish, just corresponds to a (generally non-abelian) gauge transformation

$$
A_{m} \rightarrow A_{m}^{\prime}=\exp [i \Theta(x)]\left(A_{m}+i \partial_{m}\right) \exp [-i \Theta(x)]
$$

One may readily confirm that the other components of $G$ are consistent with these changes.

This metric can be generalized further to include a Higgs scalar field by replacing the vielbein component $E_{\mu}^{\alpha}=\Lambda \delta_{\mu}^{\alpha}$ by $\Lambda \chi_{\mu}^{\alpha}$, whereupon the Higgs field resides in the property metric components $G_{\zeta \bar{\zeta}}$ as $\Phi_{\bar{\mu} \nu}=\chi_{\bar{\mu}}^{\bar{\alpha}} \delta_{\bar{\alpha} \beta} \chi_{\nu}^{\beta}$. As well one can contemplate making a supergauge property transformation where $\Theta$ depends on $\zeta, \bar{\zeta}$ too. Leaving aside these elaborations and having satisfied ourselves that gauge variations arise painlessly in this framework, we now turn to general relativistic aspects and curvatures in particular. To that end and to avoid confusion between particle physics and Einstein notations we force ourselves to writing everything in terms of real coordinates $\xi, \eta$ rather than complex $\zeta$.

\section{Curvature contributions in space-time-property}

A compact description of general relativity of space-time-property is summarised in the following Appendix, with precise orderings and sign factors which it is perilous to ignore. For the remainder of this section we will avoid the intricacies that full $\mathrm{SU}(N)$ can cause, by restricting the argument to a single complex $\zeta$ or real pair $\xi, \eta$; the only relevant group becomes $\mathrm{U}(1)$ and there is but one gauge field. What follows is therefore a mere 
skeleton of the full theory, but even so there are some very interesting features that show up.

Our first model is one which contains no gauge fields; the metric is curved in spacetime and property separately, there being no inter-twining of the two sectors. We omit property indices $\mu, \nu$, labelling them $\xi, \eta$.

$$
\begin{aligned}
d s^{2} & =d x^{m} d x^{n} G_{n m}(x, \xi, \eta)+2 i d \xi d \eta G_{\eta \xi}(x, \xi, \eta) \\
& \equiv d x^{m} d x^{n} g_{n m}(x)(1+i f \xi \eta)+2 i \Lambda^{2} d \xi d \eta(1+i g \xi \eta)
\end{aligned}
$$

incorporates curvature in property through the 'coupling constants' $f, g$, aside from the curvature due to the normal gravitational field $g_{m n}$. (Inclusion of the $\xi \eta$ pieces is very necessary to obtain non-zero results after property integration.)

The curvature components in space-time $R_{j k l m}$ are just the usual ones multiplied by the factor $(1+i f \xi \eta)$. The new ones are connected with property. We may calculate them from first principles by spelling out the metric components,

$$
\begin{gathered}
\left(\begin{array}{ccc}
G_{m n} & G_{m \xi} & G_{m \eta} \\
G_{\xi n} & G_{\xi \xi} & G_{\xi \eta} \\
G_{\eta n} & G_{\eta \xi} & G_{\eta \eta}
\end{array}\right)=\left(\begin{array}{ccc}
g_{m n}(1+i f \xi \eta) & 0 & 0 \\
0 & 0 & -i \Lambda^{2}(1+i g \xi \eta) \\
0 & i \Lambda^{2}(1+i g \xi \eta) & 0
\end{array}\right), \text { so } \\
\left(\begin{array}{ccc}
G^{l m} & G^{l \xi} & G^{l \eta} \\
G^{\xi m} & G^{\xi \xi} & G^{\xi \eta} \\
G^{\eta m} & G^{\eta \xi} & G^{\eta \eta}
\end{array}\right)=\left(\begin{array}{ccc}
g^{l m}(1-i f \xi \eta) & 0 & 0 \\
0 & 0 & -i(1-i g \xi \eta) / \Lambda^{2} \\
0 & i(1-i g \xi \eta) / \Lambda^{2} & 0
\end{array}\right),
\end{gathered}
$$

and evaluating the connections.

The non-zero ones in the property sector are

$$
\Gamma_{\xi \eta}^{\xi}=-\Gamma_{\eta \xi} \xi^{\xi}=i g \xi, \quad \Gamma_{\xi \eta}^{\eta}=-\Gamma_{\eta \xi}{ }^{\eta}=i g \eta
$$

from which one may derive the curvature components. The relevant ones are

$$
\begin{aligned}
& R_{\xi \eta \eta}^{\eta}=-2 i g(1+i g \xi \eta)=-R_{\eta \xi \xi}^{\xi} \\
& R_{\xi \xi \eta}^{\xi}=-i g(1+i g \xi \eta)=-R_{\eta \eta \xi}^{\eta}
\end{aligned}
$$

We find that the Ricci tensor components $R_{\eta \eta}, R_{\xi \xi}=0$ (obvious from antisymmetry anyway) and

$$
R_{\xi \eta}=-R_{\eta \xi}=3 i g(1+i g \xi \eta)
$$

Consequently the total curvature is given by

$$
R=G^{m n} R_{n m}+2 G^{\eta \xi} R_{\xi \eta}=R^{(g)}(1-i f \xi \eta)-6 g / \Lambda^{2} .
$$

Since $\sqrt{-G . .}=-i \Lambda^{2} \sqrt{-g . .}(1+2 i f \xi \eta)(1+i g \xi \eta)$, we obtain an action

$$
I \equiv \frac{1}{2 \Lambda^{4}} \int R \sqrt{G . .} d^{4} x d \eta d \xi=\frac{1}{2 \kappa^{2}} \int d^{4} x \sqrt{-g . .}\left[R^{(g)}+\lambda\right]
$$

where $\kappa^{2} \equiv 8 \pi G_{N}=\Lambda^{2} /(f+g), R^{(g)}$ is the standard gravitational curvature and $\lambda=6 g(2 f+g) / \Lambda^{2}(f+g)$ corresponds to a cosmological term. However the scales of 
the two parts are widely different suggesting that the coupling $g$ is incredibly miniscule. Hence this model sheds no light on this perennial problem of physics; it merely indicates that the cosmic contribution might conceivably be connected with the curvature of internal property space.

Our second example leaves property space flat (in the $\eta, \xi$ sector) but links that sector with space-time through the $\mathrm{U}(1)$ gauge field $A$. It is governed by the metric

$$
\begin{gathered}
\left(\begin{array}{ccc}
G_{m n} & G_{m \xi} & G_{m \eta} \\
G_{\xi n} & G_{\xi \xi} & G_{\xi \eta} \\
G_{\eta n} & G_{\eta \xi} & G_{\eta \eta}
\end{array}\right)=\left(\begin{array}{ccc}
g_{m n}(1+i f \xi \eta)+2 i \Lambda^{2} \xi A_{m} A_{n} \eta & i \Lambda^{2} A_{m} \xi & i \Lambda^{2} A_{m} \eta \\
i \Lambda^{2} A_{n} \xi & 0 & -i \Lambda^{2} \\
i \Lambda^{2} A_{n} \eta & i \Lambda^{2} & 0
\end{array}\right), \text { so } \\
\left(\begin{array}{ccc}
G^{l m} & G^{l \xi} & G^{l \eta} \\
G^{\xi m} & G^{\xi \xi} & G^{\xi \eta} \\
G^{\eta m} & G^{\eta \xi} & G^{\eta \eta}
\end{array}\right)=\left(\begin{array}{ccc}
g^{l m}(1-i f \xi \eta) & A^{l} \eta & -A^{l} \xi \\
-A^{m} \eta & 0 & -i\left(1 / \Lambda^{2}-i \xi A . A \eta\right) \\
A^{m} \xi & i\left(1 / \Lambda^{2}-i \xi A . A \eta\right) & 0
\end{array}\right) .
\end{gathered}
$$

Knowing full well that inclusion of $g_{m n}$ will produce generally covariant expressions, we can simplify the analysis somewhat by going to flat Minkowski space first as there are then fewer connections to worry about. With a bit of work we find $\left(F_{m n} \equiv A_{m, n}-A_{n, m}\right)$,

$$
\begin{aligned}
& \Gamma_{\xi \eta}{ }^{\xi}=\Gamma_{\xi \eta}^{\eta}=\Gamma_{\xi \eta}{ }^{k}=0, \\
& \Gamma_{m \xi}{ }^{\xi}=\Gamma_{m \eta^{\eta}}=i \Lambda^{2} A^{l} F_{l m} \xi \eta / 2, \quad \Gamma_{m \xi}{ }^{\eta}=-\Gamma_{m \eta}{ }^{\xi}=A_{m} \\
& \Gamma_{m \xi}{ }^{l}=i \Lambda^{2} F^{l}{ }_{m} \xi / 2, \quad \Gamma_{m \eta}{ }^{l}=i \Lambda^{2} F_{m}^{l}{ }_{m} \eta / 2 \\
& \Gamma_{m n}{ }^{\xi}=-A_{m} A_{n} \xi-\left(A_{m, n}+A_{n, m}\right) \eta / 2, \\
& \Gamma_{m n}{ }^{\eta}=-A_{m} A_{n} \eta+\left(A_{m, n}+A_{n, m}\right) \xi / 2, \\
& \Gamma_{m n}{ }^{k}=i \Lambda^{2}\left(A_{m} F_{n}^{k}+A_{n} F^{k}{ }_{m}\right) \xi \eta .
\end{aligned}
$$

The other Christoffel symbols are obtained through symmetry of indices.

Referring to eqns (23)-(26), one computes

$$
\begin{aligned}
& R_{k m}=R_{k l m}^{l}-R_{k \xi m}^{\xi}-R_{k \eta m}^{\eta}=-i \Lambda^{2}\left(A_{k, l}+A_{l, k}\right) F_{m}^{l} \xi \eta / 2+\text { total derivative } \\
& R_{k \xi}=R_{k l \xi}^{l}+R_{k \xi \xi}^{\xi}+R_{k \eta \xi}^{\eta}=i \Lambda^{2}\left[F_{k, l}^{l} \xi / 2+A^{l} F_{k, l} \eta\right]+\text { total derivative } \\
& R_{k \eta}=R_{k l \eta}^{l}+R_{k \xi \eta}^{\xi}+R_{k \eta \eta}^{\eta}=i \Lambda^{2}\left[F_{k, l}^{l} \eta / 2-A^{l} F_{k, l} \xi\right]+\text { total derivative } \\
& R_{\xi \eta}=\Lambda^{4} F_{k l} F^{l k} \xi \eta / 4 .
\end{aligned}
$$

The above expressions are readily covariantized by including the gravitational component $g_{m n}(1+i f \xi \eta)$. In that manner we end up with the total curvature:

$R=G^{m n} R_{n m}+2 G^{m \xi} R_{\xi m}+2 G^{m \eta} R_{\eta m}+2 G^{\eta \xi} R_{\xi \eta} \rightarrow R^{(g)}(1-i f g \xi \eta)+i \Lambda^{2} g^{k m} g^{l n} F_{k l} F_{n m} \xi \eta / 2(18)$

which is nothing more than the sum of the electromagnetic Lagrangian and the gravitational curvature. We end up with

$$
\int R \sqrt{-G . .} d^{4} x d \eta d \xi / 2 \Lambda^{4}=\int d^{4} x \sqrt{-g . .}\left[R^{(g)} / 2 \kappa^{2}-F^{k l} F_{k l} / 4\right],
$$

where $\kappa^{2}=\Lambda^{2} / f=8 \pi G_{N}$. It is a nice feature of the formalism that gauge field Lagrangian arises from space-property terms; in that respect it is quite similar to the 
standard K-K model (which comes from the tie-up between ordinary space-time and the fifth dimension).

At this point one may contemplate some generalizations. First of all it is clear that one may replace the two 'couplings' $f$ and $g$ by two distinct scalar fields (rather like dilatons), whose expectation values are $f, g$. This ought to produce some new interactions and kinetic terms involving them. Secondly, one may combine the two models above and arrive at a model of QED plus gravity plus a cosmological contribution. Thirdly, one may extend the whole enterprise to the five $\zeta$ in order to achieve a general relativistic picture of fundamental particles, though it is still a mystery (as it is in the standard picture) why the weak SU(2) subgroup should act solely on lefthanded components; possibly a right-left symmetric scheme, with spontaneous breaking of parity, might be a solution. Anyhow all of these scenarios are for future investigation and there are plenty of lessons we can learn from researches in GUTs over the last decades. What is certain is that nonlinear transformations between space and property, all the time respecting spin-statistics, are very rich and should repay study.

\section{Appendix A - Extended General Relativity}

This appendix contains our notation and definitions and is crucial because our framework turns out to be a compromise between the Einstein-Grossmann notation for general relativity and the conventional particle physics up-down description of unitary group representations. It is really important to get the factors and the order of the indices correct before we apply the ideas to a particular metric; the signs are not obvious nor are they trivial. Essentially we are rederiving general relativity for systems which contain commuting and anticommuting coordinates in quick time here...

Begin with a pair of real anticommuting coordinates $\xi, \eta$ associated with an $\mathrm{Sp}(2)$ group. Notice that their product is $\mathrm{Sp}(2)$ invariant but antihermitian since $(\xi \eta)^{\dagger}=\eta^{\dagger} \xi^{\dagger}=\eta \xi=-\xi \eta$. For this pair it is convenient to define a complex coordinate and its adjoint via $\zeta=(\xi+i \eta) / \sqrt{2}, \quad \bar{\zeta}=(\xi-i \eta) / \sqrt{2}$, whence we see that the product $\bar{\zeta} \zeta=-i \eta \xi$ is properly hermitian. Also the $\mathrm{O}(2)$ coordinate rotation $\xi \rightarrow(\xi \cos \theta+\eta \sin \theta), \quad \eta \rightarrow(-\xi \sin \theta+\eta \cos \theta)$ gets transcribed into a $\mathrm{U}(1)$ transformation $\zeta \rightarrow \mathrm{e}^{-i \theta} \zeta, \quad \bar{\zeta} \rightarrow \bar{\zeta} \mathrm{e}^{i \theta}$. So far as integration is concerned, we adopt the Berezin convention that $\int(d \zeta d \bar{\zeta})(\bar{\zeta} \zeta)=1$. This process can be continued with every $\mathrm{Sp}(2)$ pair, leading us to a set of $N$ complex anticommuting parameters labelled with up-indices like the usual spacetime ones: $\zeta^{\mu} \equiv\left(\xi^{\mu}+i \eta^{\mu}\right) / \sqrt{2}, \mu=1 \ldots N$, inviting us to construct a $\mathrm{U}(N)$ group. [In the text we have chosen $N=5$ for good reason.] If we wished to conform to the general relativistic convention we would have to write the adjoint coordinates as $\left(\zeta^{\mu}\right)^{\dagger}=\zeta^{\bar{\mu}}$ again with up-indices (and in contradistinction to the particle physics notation). Polynomials in $\bar{\zeta}$ and $\zeta$ lead to a particular set of $\mathrm{U}(N)$ representations which are examined in the main body of the article. However it is safer and certainly less confusing to adopt the real anticommuting coordinates particularly if we want to enlarge spacetime coordinates $x^{m}$ by appending property coordinates; in 
that way we obtain a super-coordinate $X^{M}=\left(x^{m}, \xi^{\mu}, \eta^{\mu}\right)$. Within such a framework the natural symmetry group involving the various $\xi, \eta$ is $\mathrm{O}(2 N)$.

We are now in a position to define a 'spacetime-property' distance which specifies not just how far apart are located the events but how different they are in character. In constructing this metrical separation we should be aware that differentiation is usually taken on the left, a convention we are obliged to adhere to. The derivative rule must thereby be expressed in the following order: $d F(X)=d X^{M}\left(\partial F / \partial X^{M}\right) \equiv d X^{M} \partial_{M} F$, not with the $d X$ on the right, and for products of functions the standard rule applies: $d(F G .)=.d F G+F d G+\ldots$ A coordinate transformation is thus described by $d X^{M}=d X^{N}\left(\partial X^{M} / \partial X^{N}\right)$, and in that particular order. With this understanding one forms a real $d s^{2}=d X^{N} d X^{M} G_{M N}$. Next we remind the reader of the standard convention of ascribing a sign factor $(-1)^{[F]}$ where $[F]=0$ when it refers to a boson and $[F]=1$ when it refers to a fermion. Thus the symmetry property of the metric is $G_{M N}=(-1)^{[M][N]} G_{N M}$. If we define the inverse by $G^{L M} G_{M N}=\delta_{N}^{L}$ then it is simple to establish that $G^{M N}=(-1)^{[M]+[N]+[M][N]} G^{N M}$.

Changing coordinate system from $X$ to $X^{\prime}$, we have to be exceedingly careful with signs and orders of products, things we normally never care about; the correct transformation law is

$$
G_{N M}(X)=\left(\frac{\partial X^{\prime R}}{\partial X^{M}}\right)\left(\frac{\partial X^{\prime S}}{\partial X^{N}}\right) G_{S R}^{\prime}\left(X^{\prime}\right)(-1)^{[N]([R]+[M])}
$$

or conversely

$$
G_{S R}^{\prime}\left(X^{\prime}\right)=\left(\frac{\partial X^{M}}{\partial X^{\prime R}}\right)\left(\frac{\partial X^{N}}{\partial X^{\prime S}}\right) G_{N M}(X)(-1)^{[S]([R]+[M])} .
$$

Transformation laws for contravariant and covariant vectors read:

$$
V^{M}\left(X^{\prime}\right)=V^{R}(X)\left(\frac{\partial X^{M}}{\partial X^{R}}\right) \quad \text { and } \quad A_{M}^{\prime}\left(X^{\prime}\right)=\left(\frac{\partial X^{R}}{\partial X^{\prime M}}\right) A_{R}(X),
$$

in the order stated. Thus the invariant contraction is

$$
V^{\prime M}\left(X^{\prime}\right) A_{M}^{\prime}\left(X^{\prime}\right)=V^{R}(X) A_{R}(X)=(-1)^{[R]} A_{R}(X) V^{R}(X) .
$$

The inverse metric $G^{M N}$ can be used to raise and lower indices as well as forming invariants, so for instance $V_{R} \equiv V^{S} G_{S R}$ and $V^{\prime R} V^{\prime S} G_{S R}^{\prime}=V^{M} V^{N} G_{N M}$. As usual these rules can be extended to tensors, so suffice it to say that $T^{L M N \text {.. }}$ will transform in the same way as a product of contravariant vectors $A^{L} B^{M} C^{N}$.., with a particular ordering of $\left(\partial X / \partial X^{\prime}\right)$ factors, etc.

The next issue is covariant differentiation but, before embarking on this, note that the well-known convention which we are obliged to respect, namely $A_{M, N} \equiv$ $\left(\partial / \partial X^{N}\right) A_{M}$ is liable to cause complications because the lower case index appears to the right of the comma but the derivative has been taken on the left! [It would have been better for our purposes if right differentiation were used but that convention is 
non-standard.] Nevertheless we shall stick to the usual requirement that $A_{M ; N}$ must transform like $T_{M N}$, viz.

$$
T_{M N}^{\prime}\left(X^{\prime}\right)=(-1)^{[S]+[N])[R]}\left(\frac{\partial X^{R}}{\partial X^{\prime M}}\right)\left(\frac{\partial X^{S}}{\partial X^{\prime N}}\right) T_{R S}(X),
$$

and see what it entails. A certain amount of work is needed to establish that

$$
A_{M ; N}=(-1)^{[M][N]} A_{M, N}-A^{L} \Gamma_{\{M N, L\}},
$$

where the connection is given by

$$
\begin{aligned}
\Gamma_{\{M N, L\}} & \equiv\left[(-1)^{([L]+[M])[N]} G_{L M, N}+(-1)^{[M][L]} G_{L N, M}-G_{M N, L}\right] / 2 \\
& =(-1)^{[M][N]} \Gamma_{\{N M, L\}} .
\end{aligned}
$$

We leave the reader to verify that this is the correct formula involving commuting and anticommuting coordinates. Another useful way to write the covariant derivative is to define

$$
\Gamma_{M N}{ }^{K} \equiv(-1)^{[L]([M]+[N])} \Gamma_{\{M N, L\}} G^{L K}=(-1)^{[M][N]} \Gamma_{N M}{ }^{K},
$$

whereupon

$$
A_{M ; N}=(-1)^{[M][N]} A_{M, N}-\Gamma_{M N}{ }^{L} A_{L} .
$$

Similarly one can show that for double index tensors the correct differentiation rule is

$$
T_{L M ; N} \equiv(-1)^{[N]([L]+[M])} T_{L M, N}-(-1)^{[M][N]} \Gamma_{L N}{ }^{K} T_{K M}-(-1)^{[L]([M]+[N]+[K])} \Gamma_{M N}{ }^{K} T_{L K}
$$

As a check on our work and sign factors it very pleasing that covariant derivative of the metric properly vanishes:

$$
G_{L M ; N} \equiv(-1)^{[N]([L]+[M])} G_{L M, N}-(-1)^{[L][M]} \Gamma_{\{L N, M\}}-\Gamma_{\{M N, L\}} \equiv 0 .
$$

We now move to the Riemann curvature tensor in its various guises. Using the above rules and definitions one can show that the difference between two successive covariant derivatives is linear in the original vector and equals

$$
A_{K ; L ; M}-(-1)^{[L][M]} A_{K ; M ; L} \equiv(-1)^{[K]([L]+[M])} R_{K L M}^{J} A_{J}
$$

where

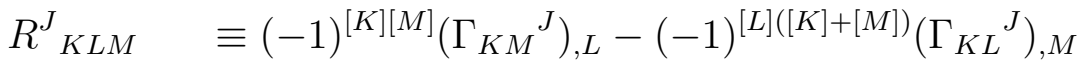

$$
\begin{aligned}
& +(-1)^{[M]([K]+[L])+[K][L]} \Gamma_{K M}{ }^{N} \Gamma_{N L}{ }^{J}-(-1)^{[K]([M]+[L])} \Gamma_{K L}{ }^{N} \Gamma_{N M}{ }^{J} \text {. }
\end{aligned}
$$

Evidently, $R_{K L M}^{J}=-(-1)^{[L][M]} R_{K M L}^{J}$ and, less obviously, the cyclical relation takes the form

$$
(-1)^{[K][L]} R_{K L M}^{J}+(-1)^{[L][M]} R_{L M K}^{J}+(-1)^{[M][K]} R_{M K L}^{J}=0 .
$$


The fully covariant Riemann curvature tensor then emerges through the contraction $R_{J K L M} \equiv(-1)^{([J]+[K])[L]} R_{K L M}^{N} G_{N J}$ and possesses the pleasing features:

$$
\begin{aligned}
R_{J K L M} & =-(-1)^{[L][M]} R_{J K M L}=-(-1)^{[J][K]} R_{K J L M}, \\
0 & =(-1)^{[J][L]} R_{J K L M}+(-1)^{[J][M]} R_{J L M K}+(-1)^{[J][K]} R_{J M K L} \\
R_{J K L M} & =(-1)^{([J]+[K])([L]+[M])} R_{L M J K} .
\end{aligned}
$$

It is then but a short step to get a suitable Ricci tensor and scalar curvature:

$$
\begin{gathered}
(-1)^{[K][M]} R_{K M} \equiv(-1)^{[J]+[K][L]+[J]([K]+[M])} G^{L J} R_{J K L M} \\
=(-1)^{[L]([K]+[L]+[M])} R^{L}{ }_{K L M}=R_{M K}, \\
R \equiv G^{M K} R_{K M} .
\end{gathered}
$$

We also anticipate that $R$ components will obey some version of the Bianchi identity. In the text we evaluate these components for particular space-time-property metrics.

\section{Appendix B - Four complex property coordinates are not enough}

Here we will explain why five property coordinates were chosen in the main body of the paper. We take it as given that one must include three colour coordinates with the same charge, having $Q=1 / 3$, if strong interactions are to be incorporated, and that the model should produce three particle generations. As well we suppose that the property of charge is additive and that other properties are obtained by taking polynomials in the coordinates $\zeta$. Another assumption is that fermions are associated with odd powers of $\zeta$. If we add just one extra $\zeta$, we must choose $Q=0$ or $Q=1$ in order to avoid outlandish charge values in the composite properties. Let's examine both cases to see where they fail.

With $Q\left(\zeta^{0}, \zeta^{1}, \zeta^{2}, \zeta^{3}\right)=(0,1 / 3,1 / 3,1 / 3)$, we may identify the following states: $\left(N, D^{c}\right) \sim\left(\zeta^{0}, \zeta^{i}\right) ; i=1,2,3$ and $\left(L^{c}, U\right) \sim\left(\zeta^{1} \zeta^{2} \zeta^{3}, \zeta^{0} \zeta^{i} \zeta^{j}\right)$, where $N, L, D, U$ stand for generic neutrino, charged lepton, down-quark, up-quark states. We thus get one generation without including the conjugate properties $\bar{\zeta}$. Incorporating the latter, another lepton emerges via the property combination $\zeta^{1} \zeta^{2} \zeta^{3} \zeta^{0} \bar{\zeta}_{0}$, and although we can get at least another two sets of $N, U, D$ there is simply no place for a third charged lepton - a pity because this model is really very economical.

With $Q\left(\zeta^{1}, \zeta^{2}, \zeta^{3}, \zeta^{4}\right)=(1 / 3,1 / 3,1 / 3,-1)$ the situation is much worse. The odd $\zeta$ sector produces two oppositely charged leptons and two sets of down-quarks: $\left(\zeta^{i}, \zeta^{4}, \zeta^{1} \zeta^{2} \zeta^{3}, \zeta^{i} \zeta^{j} \zeta^{4}\right) \sim\left(D^{c}, L, D^{\prime}, L^{\prime c}\right)$; that's not a lot of good as we are missing the neutrinos and up-quarks! Only by allowing even powers of $\zeta$ can we recover those missing states, but that's at the price of incorrect statistics. We can of course postulate a new fermionic supermultiplet which, in its even powers, contains other batches of fermions (the neutrinos and up-quarks) but this doubled viewpoint is, to our mind, an ugly extension; it is far simpler and more elegant to attach another $\zeta^{0}$.

Thus we suggest that the only proper way round these difficulties is to take five complex $\left(\zeta^{0}, \zeta^{1}, \zeta^{2}, \zeta^{3}, \zeta^{4}\right)$ with charges $(0,1 / 3,1 / 3,1 / 3,-1)$. We ascribe fermion number 
$F=(1,-1 / 3,-1 / 3,-1 / 3,1)$ to these properties to agree with standard choices, and will again assume that $F$ is additive like charge. [Of course $Q$ and $F$ are reversed for the conjugate $\bar{\zeta}$.] This does produce an abundance of particles states which we are obliged to prune, as we do in the next appendix, but the main point is that the extra number is circumscribed, unlike models which rely upon excitations around another bosonic dimension.

\section{Appendix C - Duality constraints}

Having settled on five $\zeta$ one is naturally led to $\mathrm{SU}(5)$ or $\mathrm{SO}(10)$ classification groups. Here we concentrate on the former viewpoint and to that end we make use of totally antisymmetric Levi-Civita tensors $\epsilon_{\rho \kappa \lambda \mu \nu}$ and $\epsilon^{\rho \kappa \lambda \mu \nu}$ with $\epsilon_{01234} \equiv 1=\epsilon^{01234}$ for raising and lowering indices in the sense of particle physics. [In sections 3 and 4 we have rewritten $\bar{\zeta}_{\mu}$ as $\bar{\zeta}^{\bar{\mu}}$ to conform to general relativistic notation.] The key point is that the "dual" of a polynomial, obtained via

$$
\left[(\bar{\zeta})^{m}(\zeta)^{n}\right]^{\times} \equiv \epsilon^{\cdot}(\bar{\zeta})^{5-n} \epsilon_{. .}(\zeta)^{5-m}
$$

has precisely the same charge and fermion number as the original polynomial and this provides a mechanism for cutting down the plethora of states. As two examples which define how the index order is to be preserved and typify the recipe,

$$
\begin{gathered}
{[1]^{\times}=\bar{\zeta}_{4} \bar{\zeta}_{3} \bar{\zeta}_{2} \bar{\zeta}_{1} \bar{\zeta}_{0} \zeta^{0} \zeta^{1} \zeta^{2} \zeta^{3} \zeta^{4}=\left(\bar{\zeta}_{\mu} \zeta^{\mu}\right)^{5} / 5 !} \\
{\left[\bar{\zeta}_{\tau} \zeta^{\rho} \zeta^{\sigma}\right]^{\times} \equiv \frac{1}{6} \epsilon^{\lambda \mu \nu \rho \sigma} \bar{\zeta}_{\lambda} \bar{\zeta}_{\mu} \bar{\zeta}_{\nu} \cdot \frac{1}{24} \epsilon_{\tau \alpha \beta \gamma \delta} \zeta^{\alpha} \zeta^{\beta} \zeta^{\gamma} \zeta^{\delta} .}
\end{gathered}
$$

The rule ensures that double dual corresponds to the identity: $\left[(\bar{\zeta})^{m}(\zeta)^{n}\right]^{\times \times}=(\bar{\zeta})^{m}(\zeta)^{n}$.

We hereby list all the duals needed for the eventual pruning operation, considering just odd polynomials which belong to fermions (although similar results can be obtained for the bosonic even polynomials). It is sufficient to take 5 as the maximum property power of $\zeta$ plus $\bar{\zeta}$ because duals populate the rest; also the conjugates can be determined from the set directly by suitable interchange of $\zeta \leftrightarrow \bar{\zeta}$ and will likewise be disregarded:

$$
\begin{aligned}
{\left[\zeta^{\nu}\right]^{\times} } & =\zeta^{\nu}\left(\bar{\zeta}_{\mu} \zeta^{\mu}\right)^{4} / 4 ! \\
{\left[\zeta^{\lambda} \zeta^{\mu} \zeta^{\nu}\right]^{\times} } & =-\zeta^{\lambda} \zeta^{\mu} \zeta^{\nu}\left(\bar{\zeta}_{k} \zeta^{\kappa}\right)^{2} / 2 ! \\
{\left[\zeta^{0} \zeta^{1} \zeta^{2} \zeta^{3} \zeta^{4}\right]^{\times} } & =\zeta^{0} \zeta^{1} \zeta^{2} \zeta^{3} \zeta^{4} \\
{\left[\left(\bar{\zeta}_{0} \zeta^{0}\right) \zeta^{4}\right]^{\times} } & =\left(\bar{\zeta}_{1} \zeta^{1} \bar{\zeta}_{2} \zeta^{2} \bar{\zeta}_{3} \zeta^{3}\right) \zeta^{4} \\
{\left[\bar{\zeta}_{0} \zeta^{1} \zeta^{2}\right]^{\times} } & =-\left(\bar{\zeta}_{3} \zeta^{3} \bar{\zeta}_{4} \zeta^{4}\right) \bar{\zeta}_{0} \zeta^{1} \zeta^{2} \\
{\left[\bar{\zeta}_{0} \zeta^{0} \zeta^{1} \zeta^{2} \zeta^{3}\right]^{\times} } & =-\bar{\zeta}_{4} \zeta^{4} \zeta^{1} \zeta^{2} \zeta^{3} \\
{\left[\bar{\zeta}_{0} \zeta^{1} \zeta^{2} \zeta^{3} \zeta^{4}\right]^{\times} } & =\bar{\zeta}_{0} \zeta^{1} \zeta^{2} \zeta^{3} \zeta^{4} \\
{\left[\bar{\zeta}_{0} \bar{\zeta}_{4} \zeta^{1} \zeta^{2} \zeta^{3}\right]^{\times} } & =\bar{\zeta}_{0} \bar{\zeta}_{4} \zeta^{2} \zeta^{3} \zeta^{4} \\
{\left[\bar{\zeta}_{0} \zeta^{0} \bar{\zeta}_{3} \zeta^{1} \zeta^{2}\right]^{\times} } & =\bar{\zeta}_{4} \zeta^{4} \bar{\zeta}_{3} \zeta^{1} \zeta^{2} \\
{\left[\bar{\zeta}_{0} \zeta^{0} \bar{\zeta}_{4} \zeta^{4} \zeta_{1}\right]^{\times} } & =\bar{\zeta}_{2} \zeta^{2} \bar{\zeta}_{3} \zeta^{3} \zeta_{1}
\end{aligned}
$$


Since a doubly charged lepton is associated with the combination $\bar{\zeta}_{4} \bar{\zeta}_{0} \zeta^{1} \zeta^{2} \zeta^{3}$, the only way to eliminate it is to invoke anti-selfduality.

\section{References}

[1] Delbourgo R, Jarvis P D, Warner R C 1991 Aust. J. Phys. 44135

Delbourgo R, Twisk S, Zhang R 1988 Mod. Phys. Lett. A3 1073

Delbourgo R and White M 1990 Mod Phys. Lett. A5 355

[2] McKane A 1980 Phys. Lett A76 22

Cvitanovic P and Kennedy AD 1982 Phys. Scripta 265

Halliday I G and Ricotta R M 1987 Phys. Lett. B193 241

Dunne G V 1989 J. Phys. A22 1719

[3] Georgi H and Glashow S L 1974 Phys. Rev. Lett. 32438

Pati J C and Salam A 1974 Phys. Rev. D10 275

Fritsch H and Minkowski P 1981 Phys. Rept. 7367

[4] Harari H 1975 Phys. Lett. B57 265

Shupe M A 1979 Phys. Lett. B86 87

Harari H and Seiberg N 1981 Phys. Lett. B102 263

[5] Jarvis P D and Dondi P H 1980 Z. Phys. C4 201

Jarvis P D and White M 1991 Phys. Rev. D43 4121 